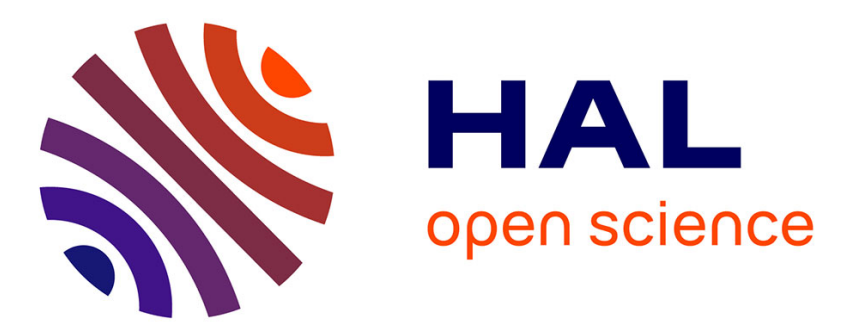

\title{
An overview of microstructural approaches for modelling and improving sound proofing properties of cellular foams: Developments and prospects
}

\author{
C. Perrot, M T Hoang, F. Chevillotte
}

\section{- To cite this version:}

C. Perrot, M T Hoang, F. Chevillotte. An overview of microstructural approaches for modelling and improving sound proofing properties of cellular foams: Developments and prospects. 10th International Styrian Noise, Vibration and Harshness Congress, Jun 2018, Gratz, Austria. hal-01818223

\section{HAL Id: hal-01818223 \\ https://hal.science/hal-01818223}

Submitted on 18 Jun 2018

HAL is a multi-disciplinary open access archive for the deposit and dissemination of scientific research documents, whether they are published or not. The documents may come from teaching and research institutions in France or abroad, or from public or private research centers.
L'archive ouverte pluridisciplinaire HAL, est destinée au dépôt et à la diffusion de documents scientifiques de niveau recherche, publiés ou non, émanant des établissements d'enseignement et de recherche français ou étrangers, des laboratoires publics ou privés. 


\title{
An overview of microstructural approaches for modelling and improving sound proofing properties of cellular foams: Developments and prospects
}

\author{
C. Perrot, M. T. Hoang, F. Chevillotte
}

\begin{abstract}
Significant advances have been made over the last 15 years in the field of modelling the acoustic properties of foams from the description of their microstructures. It entails a multidisciplinary work at the junction between physico-chemistry and mechanics of porous media, which involves a dialogue between different disciplines and requires the joint development of several techniques (imaging, upscaling, numerical computations, and experimental identification). It seems to be of timely interest to take stock of the methodological developments that have provided guidance on how to manufacture the new generation of foams with enhanced properties and to identify possible future methodological developments.
\end{abstract}

\section{Introduction}

The general objective of our work is to estimate the long-wavelength acoustical macro-behavior of real porous media from a local geometry model, a three-dimensional idealized periodic unit-cell representative of the random microstructure [1]. Attention is focused on solid foams (which are of great interest for transportation and building industries). This research is situated at the junction between three different domains: structure, properties, and manufacturing (Fig. 1). Material sciences provide most of the local geometry models and microstructure data. Engineering mechanics and to some extent statistical physics develop the main tools for multi-scale analysis of physical properties such as permeability from which part of the acoustical macro-behavior can be deduced. Physicochemical and manufacturing processes play a major role in the final product and its applications and the principal levers of microstructure modifications through manufacturing processes must be identified not only to understand and predict material properties but also in view of their improvements. The approach presented in this paper may be extended to other families of porous media such as fibrous or granular materials and the presented results generalized.

This core of the communication is based on previous papers which have been selected to exemplify the research carried out. It should represent, in a rather compact form, the main aspects of the methodology which has been used to achieve the general objective.

For the sake of understanding, we proceed by a progressive increase in complexity. First, three-dimensional idealized geometry and transports provide most of the visco-thermal dissipative phenomena appropriate for modeling sound absorbing materials [2]. Second, the solid matrix is allowed to be deformed under small perturbations to account for the effective elastic properties; an essential feature for Page 1 of 7 sound insulating simulations [3]. Direct extensions of this work could deal with an improvement of local geometry modeling (cell size elongation or degree of anisotropy, description of the struts and membranes by Plateau borders). But most real advances are obviously related to the advancement in each of its principal components. Therefore, modeling advanced geometries or providing optimal configurations should be accompanied with simultaneous improvements in the characterization techniques (elastic properties of the base material, components of the permeability tensor) and manufacturing processes (better control of the mold temperatures to suppress skin or tailoring gradient effects). This could hardly be done without reinforcing collaborations between researchers from various disciplines and groups.

An important aspect of research is that relationships between microstructure and macroscopic properties have been established, as far as possible, without any adjustable constant. This provides us with a firm framework of analysis, where the representativity of the local geometry model can be checked directly from microscopy, and each predicted macroscopic property validated from measurements (when it is not an input data) with a clear physical interpretation. This was a compulsory condition for the success of this approach. This firm link is very useful, since it stimulates general discussion, questions, remarks, criticism which greatly benefit the advancement of acoustic materials understanding.

The design of lightweight structures aims, above all, at an optimal compromise between structural properties, transport, and acoustical performances which should be compatible with some industrial requirements. Studies on the way to address the Representative Elementary Volume (REV) issue of a porous medium, which provide the basis for conducting optimization analyses, can be considered in the context of three basic categories: cellular materials, homogenization, and mathematical morphology.

Cellular materials were the focus of much attention during the past decades [4],[5]. By considering elementary cells, material scientists have produced insight into mechanical properties of porous materials, revealing how idealized building blocks of real materials are useful to derive scaling laws whose coefficients might be identified experimentally. This heritage, combined with theoretical results originating from fluid mechanics [6] have interested acousticians because they can be reexamined in an attempt to identify fruitful micro-macro, or more precisely macro-micro forms of equations relating porous materials density with a first approximation of their mechanical and transport properties [7]-[10] - a prerequisite to the optimization of acoustical performances. The great advantage of this approach is to propose simple macro-micro relationships which can 
be incorporated into complete vibro-acoustic finite element codes relaying on the Biot theory [11]. An illustration of such an approach is provided by recent works where the foam is no longer placed in a layer of its own but rather distributed in the core of the panel using topology optimization [12]. This approach is however limited by the fact that the trends suggested by numerical analysis can lead to nonmanufacturable foams. It is therefore crucial to develop relationships between microstructure and macroscopic properties of porous materials accompanying manufacturing processes. To extend further the scaling laws to foams whose closed pore content might be significant, some semi-empirical studies by Doutres et al. used coefficients that were fitted with a number of experiments [13],[14]. Such laboratory measurements are of indisputable value; however, their usefulness may be limited to a specific range of available materials already manufactured. Importantly, such curve fittings cannot reflect what are the microphysical origins behind coefficient modifications; suggesting that this approach is not the most appropriate for developing innovative materials.

In contrast, a second category of studies, which is based on numerical homogenization method, is assumed to represent the essential physics at the pore scale providing a scale separation, and was associated specifically with PUCs whose geometry is enriched as desired. Thus, research activity in this area may reflect specific attention for a particular shape, from purely open cells [15] to situations where a variable closure rate of membranes was employed to reveal those microphysical effects associated with transport phenomena [16] and linear elasticity [3]. The link between micro and macro scales is provided by the theoretical results obtained from the homogenization of periodic structures' technique; indicating the boundary value problems to be solved at micro-scale, the structure of governing equations at macro-scale, and how the associated coefficients should be computed (macroscopic properties) [17]-[19]. Semi-

phenomenological models may be used in order to compute only the asymptotic transport parameters [20], instead of the overall dynamic response functions of the porous medium - frequency by frequency [21]-[23]. Such PUCs, as expected, represent both realistic and parametrizable salient features [2],[24] of the real microstructure, allowing cellular morphology modifications to be followed, from the scale of the local geometry to the one of the engineer, where the applications on acoustics take place [25]. This builds up an appropriate framework of discussion with chemists. At the present time, however, there was no optimal morphological configuration rising from this category which was shown to be manufactured. This could be attributable to the difficulty of gathering people from disciplines and scientific cultures usually disjoined.

The third category which can be encountered when studying the REV of a porous material is generally characterized by advanced imaging techniques [i.e., micro computed tomography $(\mu \mathrm{CT})]$ associated with statistical tools originating from mathematical morphology [26] used in order to generate a porous sample statistically identical to the real one such that the disordered nature of porous media is preserved [27]-[29]. This leads to more realistic structures with a detailed description of the real medium. But because the structures are more realistic they may also be actually more difficult to parametrize, so that it is hardly possible to examine how microstructural features are related to acoustical performances and to clearly identify the optimization levers.

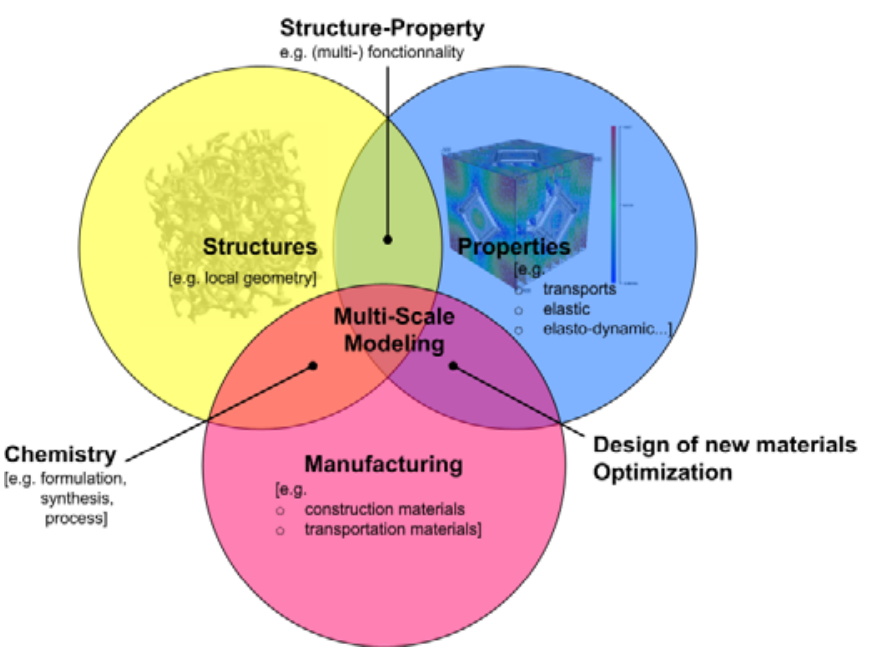

Figure 1. A research situated at the junction between three different domains.

\section{Materials and Method}

Cellular structures may be reticulated or closed cell, or somewhere between depending on formulation, processing and post-processing operations [30].The physical properties of foams depend in a complex way on many factors, some of which cannot be varied independently. Material factors include: the composition of the base material, formulation and cell structure. The majority of cellular polymers result from the nucleation, growth and expansion of gas bubbles in a melt or reacting liquid system. Representations of idealized structures corresponding to different manufacturing processes are given in Fig. 2. If the expansion process is allowed to continue the bubbles touch and distort to fill the interstrices, forming, in the three-dimensional case, polyhedral structures which are often represented in the literature by the idealized form of a Kelvin cell.
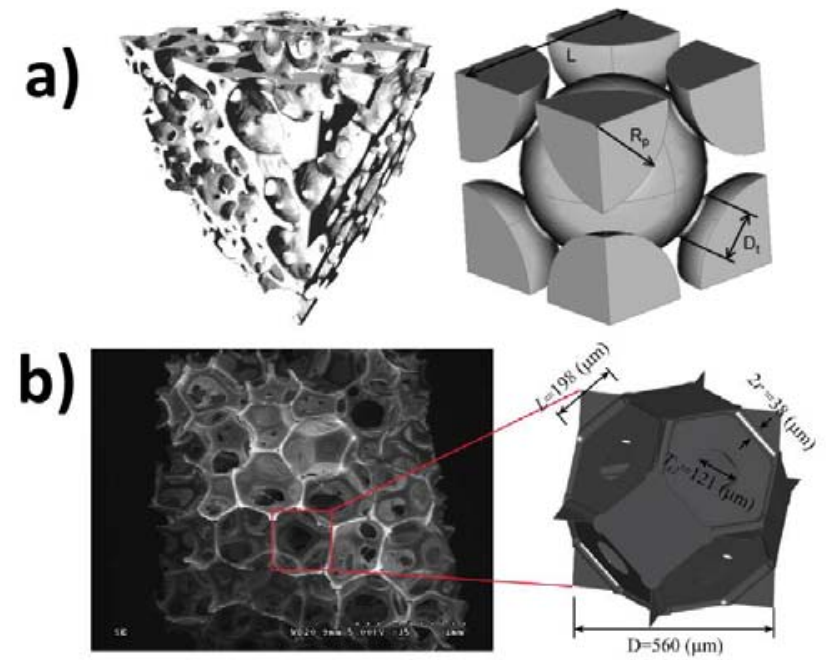

Figure 2. Examples of periodic unit cell (PUC) identifications. a) The manufacturing process involves gas injection into a solid matrix. The identified PUC is a body-centered cubic (BCC) unit cell. b) In the second example, a polyurethane foam is examined, and the identified PUC is a Kelvin cell.

Page 2 of 7 
The membranes between the cell struts which result from the intersection of the spherical bubbles (the 'windows') may be ruptured. In the case of polymeric foams this can be achieved by means of a chemically based cell-opening mechanism or by post-processing treatment (reticulation) [31].

When an acoustic wave propagates through an air saturated porous media having a motionless and isothermal skeleton, the frequencydependent interplay between viscosity and inertia, and thermal conduction produce a spatial gradient of fluid velocity and temperature; and the shear combined with heat transfer between successive layers of fluid causes compression wave attenuation [32]. The characteristic lengths of the compression waves created by an oscillating body in which the main visco-inertial and thermal dissipation processes occur are the viscous and thermal boundary layers. They respectively scale as $(v / \omega)^{1 / 2}$ and $\left(v^{\prime} / \omega\right)^{1 / 2}$, in which $v=$ $\eta / \rho_{0}$ is the fluid's cinematic viscosity, $v^{\prime}=\kappa / \rho_{0} C_{p}$ is its thermal diffusivity, $\omega$ is the angular frequency of motion; and where $\eta$ is the fluid's dynamic viscosity, $\rho_{0}$ is its density at rest, $\kappa$ is its thermal conductivity, $\mathrm{C}_{\mathrm{p}}$ is the specific heat at constant pressure. For small perturbations, because the wavelength $\lambda$ of the incident compression wave greatly exceeds the typical pore size $D$ of the motionless porous media at audible frequencies, visco-inertial motions and thermal conduction can be decoupled within a pore. The viscous forces at low frequencies (Stokes flow), inertial forces at high frequencies (potential flow formally identical to electric conduction), and thermal conduction at low frequencies (similar to diffusion-controlled reactions) together determine all the transport parameters within a porous material.

On the other hand, the elastic behavior of the solid matrix is subsequently incorporated as an additional pressure source term for the fluid flow to recover the Biot-Allard theory from first-principle calculations. The effective mechanical properties are obtained numerically for different solid foam models from finite element calculations and may be compared to values coming from experiments on real samples. The effective linear-elastic properties of the solid networks are determined by a finite element scheme operating on discretized representations of the structure; see [3] for further details. The material constituting the skeleton is assumed to be locally isotropic and linear-elastic; the elastic properties of the gaseous fluid phase are considered as negligible compared to the ones of the solid skeleton.

The effective elastic longitudinal modulus $E_{L}$ and Poisson ratio $v_{L}$ are obtained by applying two macroscopic external strains on the cube that bounds the solid foam model: a tensile strain and a shear strain related to the main coordinate directions. Since the selected solid foam models have cubic symmetry, three independent elastic constants $C_{11}, C_{22}$ and $C_{44}$ (in contracted notations) exist in the elasticity matrix, whose identification requires two numerical experiments using periodic boundary conditions, to express $E_{L}$ and $v_{L}$. The effective elastic longitudinal modulus $E_{L}$ and Poisson ratio $v_{L}$ are obtained by applying the $E_{L}=\left(C_{11}{ }^{2}+C_{11} C_{12}-2 C_{12}{ }^{2}\right) /\left(C_{11}+C_{12}\right)$ and $v_{L}=C_{12} /\left(C_{11}+C_{12}\right)$ formulas which are valid for the effective elastic properties along the principal coordinate directions, [100], [010] or [001] of materials with cubic symmetry.

The real porous frame does not have the cubic symmetry. On the contrary, real foams are either isotropic or transversely isotropic, the isotropy plane being orthogonal to the growing direction of the foam during the manufacturing process. However, the main idea is that "locally", the elastic properties of the material are correctly represented by a cellular model which exhibits cubic symmetry. The properties of "effective" materials having isotropic or transversely isotropic properties can be obtained by considering that the local cubic cells can have random orientations. Following this idea, the effective elastic properties of an effective transversely isotropic material (resp. of an effective isotropic material) correspond to the properties obtained by random orientation of the unit cell when using arbitrary rotation around a given axis (resp. when using any arbitrary rotation in space). It should be interesting to use the average of effective properties corresponding to different orientations of the unit cell. As is well known, this average corresponds to bounds on the effective properties. Thus, two bounds on the effective properties can be obtained, one given by averaging the elasticity tensor (components $C_{i j}$ ) the other by averaging the compliance tensor (components $S_{i j}$ ). Due to the fact that properties corresponding to different orientations display a moderate contrast, these two bounds are close and in the following procedure, only the bound related to the average of compliances will be used. The detailed expression obtained by this angular averaging procedure yields for the isotropic case a lower bound for the Young's modulus of the isotropic material: $E_{I}=\left[5\left(C_{11}-\right.\right.$ $\left.\left.C_{12}\right)\left(C_{11}+2 C_{12}\right) C_{44}\right] /\left[C_{44}\left(3 C_{11}+C_{12}\right)+2\left(C_{11}-C_{12}\right)\left(C_{11}+2 C_{12}\right)\right]$, and an estimate of the Poisson ratio $v_{I}=\left[-\left(C_{11}-3 C_{12}\right) C_{44}+\left(C_{11}-C_{12}\right)\left(C_{11}\right.\right.$ $\left.\left.+2 C_{12}\right)\right] /\left[C_{44}\left(3 C_{11}+C_{12}\right)+2\left(C_{11}-C_{12}\right)\left(C_{11}+2 C_{12}\right)\right]$.

\section{Results and Discussions}

\section{Microstructural optimizations and sound absorbing properties}

The sound absorption average (SAA) rating is analyzed as a function of the porosity $\phi$ and the pore radius Rp (Figure 2). The resulting 2D maps and the corresponding $\mathrm{SAA}_{125-4000}$ ratings are presented in Fig. 3 for the diffuse field (DF) excitations with a thickness of $25 \mathrm{~mm}$. The thick line shows the morphological configurations maximizing the sound absorption rating $\mathrm{SAA}_{125-4000}$. The contours enabling $95 \%$ of the maximum performance are also shown in these maps.

The calculated configurations maximizing the sound absorption rating $\left(\mathrm{SAA}_{125-4000}\right)$ for thicknesses between 10 and $50 \mathrm{~mm}$ are also given for the DF excitation (see Fig. 4). The zones having a rating higher than $95 \%$ of the maximum SAA are also illustrated for each thickness. The value of $95 \%$ is taken for each thickness using the reference of the maximum SAA for the considered thickness.

Finally, the corresponding specific throat radii, maximizing the sound absorption rating, are plotted as a function of the open porosity for thicknesses between 10 and $50 \mathrm{~mm}$ for the DF excitation in Fig. 5. The reported specific throat radii are increasing with the sample thicknesses whatever the considered range of open porosities [0.70.99]. More thorough explanations of the use of these graphs are given below using practical examples.

As shown in Figs. 4 and 5, the melamine foam, known as a good sound absorber, with parameters $\phi=0.99 ; \mathrm{Rp} \sim 100 \mu \mathrm{m}$; Rt $\sim 50 \mu \mathrm{m}$ has the structure which is optimized for high sound absorption under $\mathrm{DF}$ and for thicknesses higher than $30 \mathrm{~mm}$ (marker M in Figs. 4 and 5 , $\mathrm{SAA}^{\mathrm{DF}}{ }_{125-4000}=28 \%$ ). The full list of parameters can be found in Table 13.1 of Ref. [33] and a picture of the morphology can be found in Fig. 3 of Ref. [34]. In contrast, the maximum in sound absorption for open cell foams that have a thickness around $10 \mathrm{~mm}$ is directly linked to microstructural characteristic lengths (i.e., pore and throat size) that must be smaller. As it can be seen from Figs. 4 and 5, an improvement of the sound absorption is expected for a structure as defined by a combined reduction of the pore and throat sizes ( $\mathrm{Rp} \sim$ $40 \mu \mathrm{m}$; Rt $\sim 20 \mu \mathrm{m}$, marker M' in Figs. 4 and 5). The single number rating $\mathrm{SAA}^{\mathrm{DF}}{ }_{125-4000}$ increases from $28 \%$ to $37 \%$ in the DF.

Page 3 of 7 


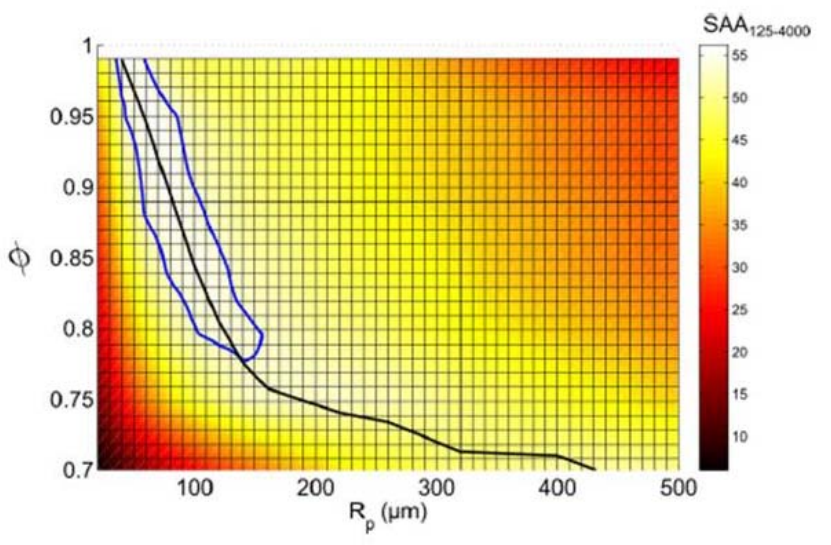

Figure 3. Effect of the open porosity $\phi$ and pore size Rp on the sound absorption rating with a diffuse field excitation. Sample thickness, $25 \mathrm{~mm}$.

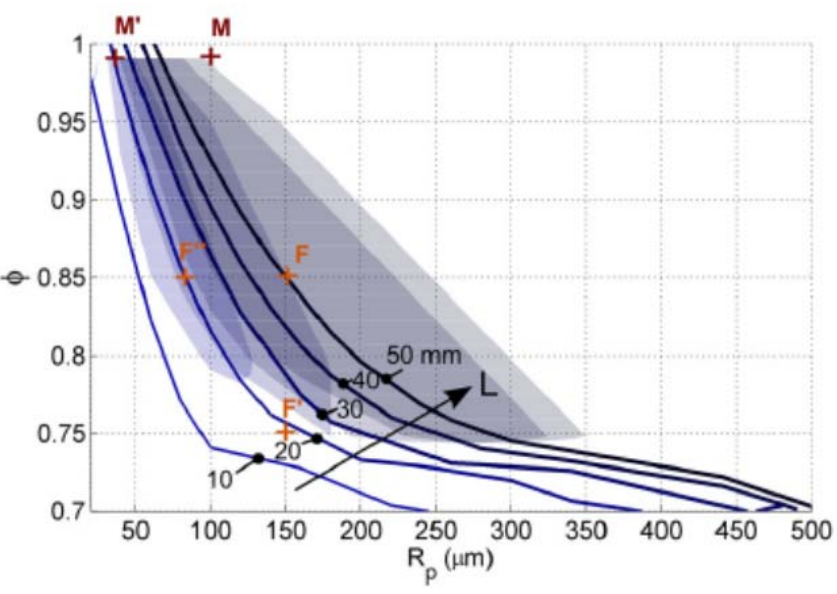

Figure 4. Effect of the open porosity $\phi$ and pore size Rp on the sound absorption rating for various sample thicknesses with a diffuse field excitation.

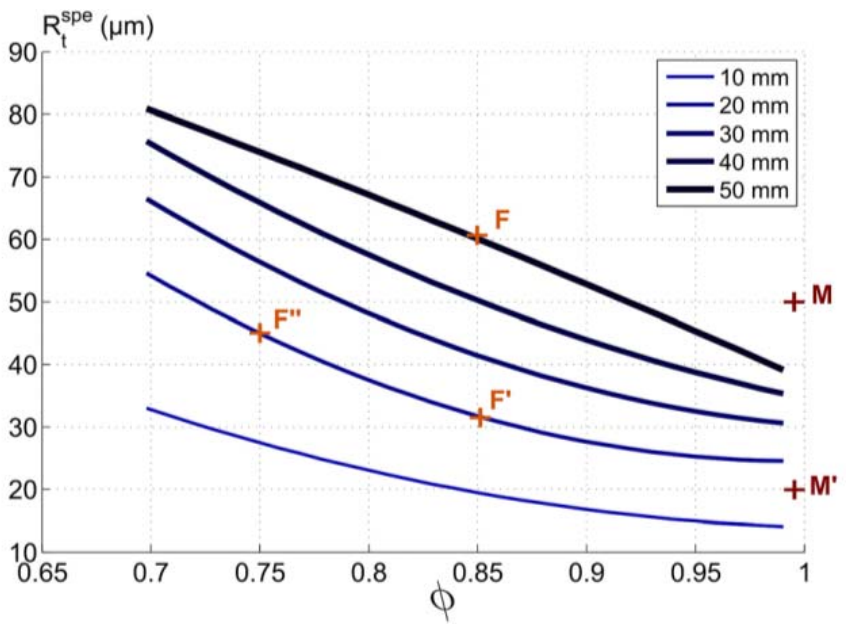

Figure 5. Specific throat radii defined as the throat radii allowing maximum sound absorption rating as a function of the open porosity for various sample thicknesses with a diffuse field excitation.
The second example is provided by foam manufactured such that the following parameters are obtained: $\phi=0.85 ; \mathrm{Rp} \sim 150 \mu \mathrm{m} ; \mathrm{Rt} \sim 60$ $\mu \mathrm{m}$ and $\mathrm{L}=20 \mathrm{~mm}$ (marker F in Figs. 4 and $5, \mathrm{SAA}^{\mathrm{DF}}{ }_{125-4000}=44 \%$ ). An analysis of the data reported in Ref. [35] reveals that there are three means to improve the sound absorption of this foam:

- The simplest way to improve the sound absorption properties of this foam is to provide it into the thickness corresponding to the best achieved rating at a given pore radius. It is shown in Fig. 4 that this kind of foam should be used with a thickness of $50 \mathrm{~mm}$ when a DF is taken as the excitation.

- If the pore size can be reduced to $\mathrm{Rp} \sim 80 \mu \mathrm{m}$, this would lead to a significant improvement of the sound absorption with a DF excitation while keeping the initial thickness of $20 \mathrm{~mm}$ and the porosity $\phi=0.85$. The throat size would be reduced to Rt $\sim 30$ $\mu \mathrm{m}$ and the single number rating $\mathrm{SAA}^{\mathrm{DF}}{ }_{125-4000}$ would reach $49 \%$ in a DF (marker F' in Figs. 4 and 5).

- Finally, the porosity could be reduced to $\phi=0.75$ to improve the sound absorption while keeping the thickness of $20 \mathrm{~mm}$ and the pore size $\mathrm{Rp}=150 \mu \mathrm{m}$. The throat size would be reduced to Rt $45 \mu \mathrm{m}$ and the single number rating SAA ${ }^{\mathrm{DF}} 125-4000$ would reach $48 \%$ in a DF (marker F' in Figs. 4 and 5).

\section{Microstructural optimizations and sound insulating properties}

In all of well-known automotive insulation solutions such as mass spring system or Light-Weight Concept (LWC: 3 layers, absorber + barrier + decoupler), the foam layer in contact with the body in white is used as a decoupler [36]. This section will focus on this decoupling function optimization. For this purpose, a pure insulation "massspring" system is studied with a heavy layer (HL) on the top of decoupling foam (Fig. 6). The heavy layer is a high density impervious material representing the "mass" element; this layer is separated from the steel via the "spring" foam to form a double-wall system (steel + foam + heavy layer). To estimate the performance of the insulator (heavy layer + foam) independently from the steel, the Insertion Loss (IL) is proposed as the difference of transmission loss (TL) between the overall system with steel and the one without steel: $I L=T L_{\text {Insulator }}+$ Steel $-T L_{\text {Steel }}$. Generally the Insertion Loss of such double-wall system increases at about $12 \mathrm{~dB} /$ octave up to the coincidence frequency when it works properly. This approximate linear increment leads this classic concept to be used efficiently in many cases to answer the insulation demand in vehicles.

This part is dedicated to defining the optimized microstructures of polyurethane (PUR) foam corresponding to its best decoupling function. For this purpose a simple system is studied with heavy layer of $3.5 \mathrm{~kg} / \mathrm{m}^{2}$ on the top of $20 \mathrm{~mm}$ soft PUR foam, this double layer is laid on a thin steel plate. A previous study was carried out by Duval et al. to set the Biot-Allard parameters target setting for insulation

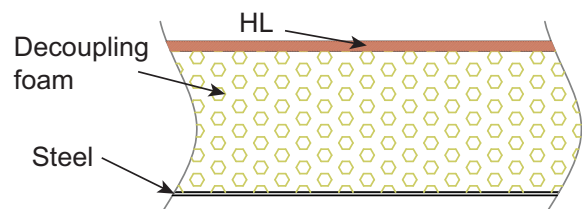

Figure 6. Classical spring foam + HL concept.

Page 4 of 7 

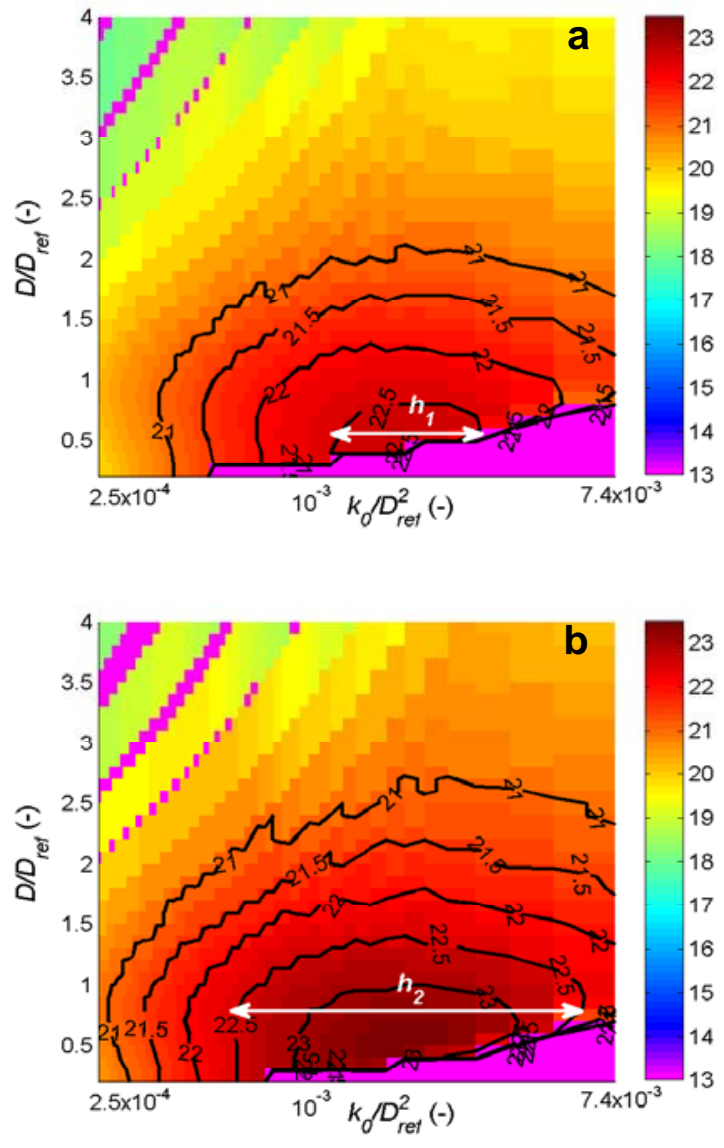

Figure 7. Insertion Loss $I L_{1000 \mathrm{~Hz}}(\mathrm{~dB})$ response surfaces corresponding to various dimensionless cell sizes $D / D_{\text {ref }}$ and dimensionless static permeabilities $k_{0} / D^{2}{ }_{r e f}$ at porosities 0.90 (a) and 0.95 (b).

problem [37]. The objective of this work was to improve the BiotAllard parameters of a so called standard soft foam by changing only one parameter at a time and by computing the corresponding Transmission Loss with the FTMM (Finite Transfer Matrix Method) simulation [33]. Then the microstructure of the foam corresponding to improved parameters could be determined by using a hybrid numerical approach [16] revealing an admissible morphological path. This latter method allowed defining a better foam microstructure but is not necessarily an optimal due to the low number of investigated cases.

Considering this limitation, an advanced numerical experiment was performed. The hybrid numerical approach applied for foam by Hoang and Perrot was used, with three input parameters: porosity, airflow resistivity, and a microstructural size such as the pore size. The elastic parameters of PUR foam were however fixed: density $\rho=$ $55 \mathrm{~kg} / \mathrm{m}^{3}$, Young's modulus $E=15 \mathrm{kPa}$. Several parametric studies are then carried out for a large range of input parameters. For each triplet of input parameters, a set of transport parameters is defined corresponding to a unique microstructure of foam. The Insertion Loss of the HL - foam system is then computed by using FTMM simulation with a spatial windowing to take into account the finite size of sample [33]. Because the simulated Insertion Loss is a frequency-dependent parameter, the Insertion Loss at $1000 \mathrm{~Hz}$, named " $\mathrm{IL}_{1000 \mathrm{~Hz}}$ ", is proposed to approximately represent the insulation performance of the insulator from the IL curve.
Following this approach, the effect of cell size $D$ is first studied. A large range of cell sizes is used which respects the pore sizes of common PUR foams. The input airflow resistivity $\sigma$ increases from a low value of about $10000 \mathrm{Nm}^{-4} \mathrm{~s}$ to a sufficient high value beyond the upper foam's airflow resistivity limit defined by Duval et al. [37]. The foam porosities $\phi$ are ranging between 0.90 and 0.99 . All parameters are rendered dimensionless via the cell-size $D_{\text {ref }}$ which is defined as the cell size of a so-called standard foam (initial foam).

Fig. 7 illustrates the $I L_{1000 \mathrm{~Hz}}$ as a function of the dimensionless static permeability $k_{0} / D_{\text {ref }}^{2}\left(k_{0}\right.$ relates to $\sigma$ through the dynamic viscosity $\eta$ of air , $k_{0}=\eta / \sigma$ ) and the dimensionless cell size $D / D_{\text {ref }}$ at a given porosity. Each point in Fig. 7(a-b) corresponds thus to a triplet of input parameters, from which a foam's microstructure together with a set of transport properties are determined. The acoustic performance defined by $I L_{1000 \mathrm{~Hz}}$ is computed thanks to these transport parameters and presented by different colors in the figure. The non-physical input parameters corresponding unrealistic microstructural configurations were suppressed (purple color).

Fig. 7 shows that the insulation level of foam depends clearly on the cell size, for example at $\phi=0.95$, the value of $D / D_{\text {ref }}$ must be inferior to 2.5 to reach a value of $I L_{1000 \mathrm{~Hz}}>21 \mathrm{~dB}$. In particular, the initial foam $\left(D / D_{\text {ref }}=1\right)$ could further be improved by decreasing the cell size down to the high performance area (better than $22.5-23 \mathrm{~dB}$ ). Regarding porosity, we note that in the range between 0.90 and 0.99 the optimal area $I L_{1000 \mathrm{~Hz}}$ varies significantly. For example, at a porosity value equal to 0.95 the high performance area is larger and about $0.5 \mathrm{~dB}$ compared to the performance area obtained with a porosity equal to 0.90 (Fig. 7(a-b), $h_{2}>h_{1}$ ). Consequently it is important to take the influence of porosity into account in the foaming process.

To investigate the influence of membrane content on the performance of the decoupling foam, the same strategy is used with the membrane closure rate $\delta / \delta_{\max }$ replacing the cell size $D$ as the input parameter. The result illustrated on Fig. 8a indicates that $\delta / \delta_{\max }$ must be inferior or equal to 1 to be in the optimal area with an $I L_{1000 \mathrm{~Hz}}$ better than 23 $\mathrm{dB}$. Using the proposed model, this means that it is preferable not to have any closed window on the real foam sample. It must be emphasized that the tortuosity strongly depends on the membrane closure rate: this parameter increases while the membrane content becomes larger. That explains why tortuosity $\alpha_{\infty}$ should not be greater than two to be in the high performance area, as illustrated on Fig. 8b. See [38] for further details.

Page 5 of 7 

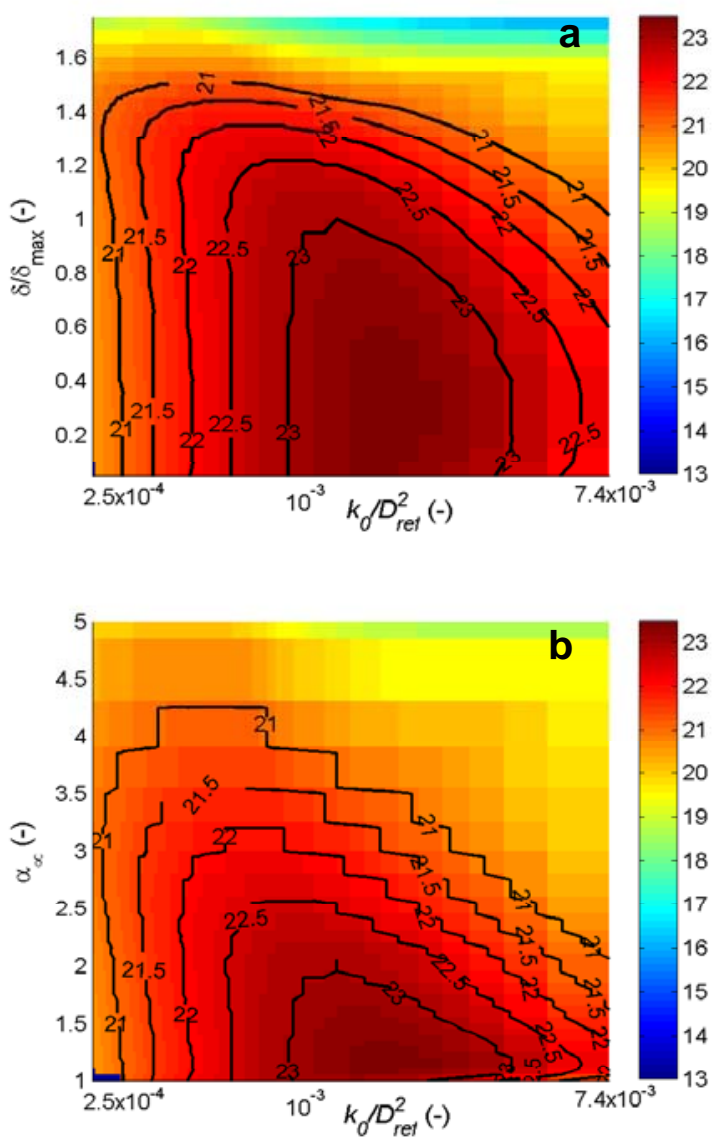

Figure 8. Insertion Loss $\mathrm{IL}_{1000 \mathrm{~Hz}}(\mathrm{~dB})$ response surfaces corresponding to various membrane closure rates $\delta / \delta_{\max }$ (a) or tortuosities $\alpha_{\infty}$ (b) and dimensionless static permeabilities $k_{0} / D^{2}$ ref at porosity 0.95 .

\section{Conclusions and Outlook}

Initially, the main concerns of the pioneering studies in this field has been to reveal whether idealized unit cells can be used to capture the main physics of visco-thermal and structural losses when sound waves are propagating through a porous media. With theoretical results from homogenization of periodic structures, imaging techniques, numerical methods and experimental validation procedures, research interests focused on revealing the microstructural configurations which can be used to effectively enhance sound proofing under the constraints of a given manufacturing process. The following critical questions have motivated several works at the junction between different disciplines. Are there periodic unit cells that can be identified as being able to emulate properly heterogeneous media (such as real solid foams of industrial interest)? Is there a direct comparison between measured and modeled characteristic sizes of the microstructure? What are the local geometry features playing a significant role in the propagation and dissipation of audible sound waves in real porous media? Important conclusions drawn from several previous studies can be summarized as (1) A direct link between the microstructure morphology and the acoustical macro-behavior can be obtained all along the multi-scale modeling process without any adjusted parameter. (2) Controlling pore and throat sizes is a useful strategy Page 6 of 7 for engineering the sound absorption spectrum and sound transmission loss of foams.

This overview has focused on recent advances in microstructural approaches for modeling and improving sound proofing properties of cellular foams. For large scale applications, low cost mass manufacturing is an inevitable step. However, 3D printing is still too expensive for mass manufacturing, and the use of the concept of optimization under an admissible manufacturing way still promising not only to better understand dissipation mechanisms but also to support the development of novel materials (composite structures, recycled materials, highly heterogeneous and hierarchical materials).

\section{References}

1. Perrot, C., "Modeling of multi-scale and multiphysical properties of acoustic materials," Habilitation thesis, Paris-Est University, 2014.

2. Hoang, M. T. and Perrot, C., "Identifying local characteristic lengths governing sound wave properties in solid foams," J. Appl. Phys. 113, 084905-7, 2013, doi: 10.1063/1.4793492.

3. Hoang, M. T., Bonnet, G., Luu, H. T., Perrot, C., "Linear elastic properties derivation from microstructures representative of transport parameters," J. Acoust. Soc. Am. 135, 3172-3185, 2014, doi: 10.1121/1.4872296.

4. Gibson, L. J. and Ashby, M. F., "Cellular solids, structure and properties," Oxford, United Kingdom, 184-192, 1988, doi: 10.1017/CBO9781139878326.

5. Warren, W. E. and Kraynik, A. M., "The linear elastic properties of open cell foams," J. Appl. Mech. 55, 341-346, 1988, doi: 10.1115/1.3173680

6. Allard, J. F. and Champoux, Y., "New empirical equations for sound propagation in rigid frame fibrous materials," J. Acoust. Soc. Am. 91, 3346-3353, 1992, doi: 10.1121/1.402824.

7. Johnson, D. L., Koplik, J., and Dashen, R., "Theory of dynamic permeability and tortuosity in fluid saturated porous media," J. Fluid Mech. 176, 379-402, 1987, doi: $10.1017 / \mathrm{S} 0022112087000727$.

8. Champoux, Y. and Allard, J. F., "Dynamic tortuosity and bulk modulus in air-saturated porous media," J. Appl. Phys. 70, 19751979, 1991, doi: $10.1063 / 1.349482$.

9. Göransson, P., "Acoustic and vibrational damping in porous solids," Phil. Trans. R. Soc. A 364, 89-108, 2006, doi: 10.1098/rsta.2005.1688.

10. Lind-Nordgren, E. and Göransson, P., "Optimising open porous foam for acoustical and vibrational performance," J. Sound and Vib. 329, 753-767, 2010, doi: 10.1016/j.jsv.2009.10.009.

11. Biot, M. A., "Theory of propagation of elastic waves in a fluidsaturated porous solid," I. Low-frequency range, J. Acoust. Soc. Am. 28, 168-178, 1956; II. Higher frequency range, J. Acoust. Soc. Am. 28, 179-191, 1956.

12. Cameron, C. J., Lind-Nordgren, E., Wennhage, P. and Göransson, P., "On the balancing of structural and acoustic performance of a sandwich panel based on topology, property, and size optimization," J. Sound and Vib. 333, 2677-2698, 2014, doi: $10.1016 /$ j.jsv.2014.01.025.

13. Doutres, O., Atalla, N., and Dong, K., "Effect of the microstructure closed pore content on the acoustic behavior of polyurethane foams," J. Appl. Phys. 110, 064901, 2011, doi: 10.1063/1.3631021.

14. Doutres, O., Atalla, N., and Dong, K., "A semiphenomenological model to predict the acoustic behavior of fully and partially reticulated polyurethane foams," J. Appl. Phys. 113, 054901, 2013, doi: 10.1063/1.4789595. 
15. Perrot, C., Panneton, R. and Olny, X., "Periodic unit cell reconstruction of porous media: Application to open cell aluminum foams," J. Appl. Phys. 101, 113538, 2007, doi: $10.1063 / 1.2745095$.

16. Hoang, M. T. and Perrot, C., "Solid films and transports in cellular foams," J. Appl. Phys. 112, 054911, 2012, doi: $10.1063 / 1.4751345$.

17. Auriault, J. L., "Dynamic behavior of a porous medium saturated by a Newtonian fluid," Int. J. Eng. Sci. 18, 775-785, 1980, doi: 10.1016/0020-7225(80)90025-7.

18. Burridge, R. and Keller, J. B., "Poroelasticity equations derived from microstructure," J. Acoust. Soc. Am. 70, 1140-1146, 1981, doi: 10.1121/1.386945.

19. Boutin, C., "Rayleigh scattering of acoustic waves in rigid porous media," J. Acoust. Soc. Am. 122, 1888-1905, 2007, doi: 10.1121/1.2756755.

20. Gasser, S., Paun, F., and Bréchet, Y., "Absorptive properties of rigid porous media: Application to face centered cubic sphere packing," J. Acoust. Soc. Am. 117, 2090-2099, 2005, doi: 10.1121/1.1863052.

21. Perrot, C., Panneton, R. and Olny, X., "Computation of the dynamic thermal dissipation properties of porous media by Brownian motion simulation: Application to an open-cell aluminum foam," J. Appl. Phys. 102, 074917, 2007, doi: $10.1063 / 1.2786899$

22. Perrot, C., Chevillotte, F. and Panneton, R., "Dynamic viscous permeability of an open-cell aluminum foam: computations vs experiments," J. Appl. Phys. 103, 024909, 2008, doi: 10.1063/1.2829774.

23. Perrot, C., Chevillotte, F., Panneton, R., Allard, J.-F et al., "On the dynamic viscous permeability tensor symmetry," J. Acoust. Soc. Am. 124, EL 210-2017, 2008, doi: 10.1121/1.2968300.

24. Perrot, C., Chevillotte, F., Hoang, M. T., Bonnet, G. et al., "Microstructure, transport, and acoustic properties of open-cell foam samples: Experiments and three-dimensional numerical simulations," J. Appl. Phys. 111, 014911, 2012, doi: 10.1063/1.3673523.

25. Perrot, C., Chevillotte, F. and Panneton, R., "Bottom-up approach for microstructure optimization of sound absorbing materials," J. Acoust. Soc. Am. 124, 940-948, 2008, doi: 10.1121/1.2945115.

26. Serra, J., "Image analysis and mathematical morphology, "Academic press, London, 1982.

27. Malinouskaya, I., Mourzenko, V. V., Thovert, J.-F., and Adler, P. M., "Wave propagation through saturated porous media," Phys. Rev. E 77, 066302, 2008, doi: 10.1103/PhysRevE.77.066302.

28. Peyrega, C., Jeulin, D., Delisée, C. and Malvestio, J., "3D morphological characterization of phonic insulation fibrous media," Adv. Eng. Mat. 13, 156-164, 2011, doi: 10.1002/adem.201000257.

29. Peyrega, C. and Jeulin, D., "Estimation of acoustic properties and of the representative volume element of random fibrous media," J. Appl. Phys. 113, 104901, 2013, doi: 10.1063/1.4794501.

30. Artavia, L.D. and Macosko, C.W., "Polyurethane flexible foam formation", (pp. 22-55); Priester, R.D. and Turner, R. B., "The morphology of flexible polyurethane matrix polymers", (pp. 78103); in Low density cellular plastics: physical basis of behavior, Edited by Hilyard, N.C. and Cunningham, A., Chapman \& Hall, London, 1994.

31. Doutres, O., Atalla, N., Brouillette, M., Hébert, C., "Using shock waves to improve the sound absorbing efficiency of closed-cell foams," Appl. Acoust. 79, 110-116, 2014, doi: 10.1121/1.4805406.

Page 7 of 7
32. Kirchhoff, G., "Über den Einfluss der Wärmeleitung in einem Gase auf die Schallbewegung," Ann. Phys. Chem. 134, 177, 1868 ["On the influence of heat conduction in a gas on sound propagation," in Physical Acoustics, edited by Lindsay, R. B., Hutchison \& Ross, Dowden, 1974]; see also Strutt, W. (Lord Rayleigh), "Theory of Sound", 2nd ed., Dover, New York, Vol II, Sec. 348, 1945; and Zwikker, C. and Kosten, C. W., "Sound Absorbing Materials", Elsevier, New York, Chapter II, 1949.

33. Allard, J. F. and Atalla, N., "Propagation of Sound in Porous Media: modeling sound absorbing materials", 2nd Ed., Wiley, Chichester, 2009.

34. Jaouen, L., Renault, A., Deverge, M., "Elastic and damping characterizations of acoustical porous materials: available experimental methods and application to a melamine foam," Appl. Acoust. 69, 1129-1140, 2008, doi: 10.1016/j.apacoust.2007.11.008

35. Chevillotte, F. and Perrot, C., "Effect of the three-dimensional microstructure on the sound absorption of foams: A parametric study," J. Acoust. Soc. Am. 142(2), 1130-1140, 2017, doi: 10.1121/1.4999058.

36. Duval, A., Rondeau, J.-F., Dejaeger, L., Lhuillier, F. et al., "Generalized Light-Weight Concepts: A New Insulator 3D Optimization Procedure," SAE Conference, p. 12., Grand Rapids (MI), 2013.

37. Duval, A., Hoang, M. T., Perrot, C., Marcel, V. et al. "Chemistry-process morphology control of porous microstructures: a bottom-up acoustic optimization approach," SIA-SFA Conference: Light-weighting and acoustical materials in vehicles, p. 8., Compiègne, France, 2013.

38. Hoang, M. T., Perrot, C., Marcel, V., Rondeau, J.-F., "Microstructure morphological optimization of foam-based lightweight insulators," Automotive NVH Comfort, Le Mans, France, October 19-20, 2016. 